\title{
VEGF Expression and Receptor Activation in the Choroid during Development and in the Adult
}

\section{Citation}

Saint-Geniez, Magali, Angel E. Maldonado, and Patricia A. D’Amore. 2006. "VEGF Expression and Receptor Activation in the Choroid During Development and in the Adult." Investigative Opthalmology \& Visual Science 47 (7) (July 1): 3135. doi:10.1167/iovs.05-1229.

\section{Published Version}

10.1167/iovs.05-1229

\section{Permanent link}

http://nrs.harvard.edu/urn-3:HUL.InstRepos:35013978

\section{Terms of Use}

This article was downloaded from Harvard University's DASH repository, and is made available under the terms and conditions applicable to Other Posted Material, as set forth at http:// nrs.harvard.edu/urn-3:HUL.InstRepos:dash.current.terms-of-use\#LAA

\section{Share Your Story}

The Harvard community has made this article openly available.

Please share how this access benefits you. Submit a story.

\section{Accessibility}




\title{
VEGF Expression and Receptor Activation in the Choroid during Development and in the Adult
}

\author{
Magali Saint-Geniez, ${ }^{1,2}$ Angel E. Maldonado, ${ }^{1,2}$ and Patricia A. D'Amore ${ }^{1,2,3,4}$
}

Purpose. Previous studies have demonstrated a role for the retinal pigment epithelium (RPE) in the development and maintenance of the choroidal vasculature, suggesting that RPE serves a trophic role for the choroidal vessels. The goal of this study was to determine the expression pattern of vascular endothelial growth factor (VEGF) and its receptors and their activation status in embryonic and adult choroid, with the purpose of providing cues regarding the role of VEGF in development and stabilization of the choroidal vasculature.

Methods. Transgenic VEGF-LacZ mice were used to examine VEGF expression in embryonic and adult eyes. Expression of VEGF isoforms and receptors in the RPE-choroid complex was assessed by RT-PCR and real-time PCR. VEGF receptor 2 expression was assessed by immunohistochemistry and its activation state was examined by immunoprecipitation followed by phosphotyrosine blot.

REsults. VEGF is expressed by RPE throughout the choroidal vascular development and in the adult. The major VEGF isoforms detected in adult RPE were VEGF120 and VEGF164, with almost no detectable VEGF188. RT-PCR analysis showed expression of VEGF receptors and coreceptors in the RPE-choroid complex. VEGFR2 was detected in the choriocapillaris underlying the RPE. Immunoprecipitation and phosphotyrosine blot of this receptor revealed that VEGFR2 is activated in adult mouse and bovine choroids.

ConcLusions. The observations suggest that VEGF signaling is involved, not only in choroidal vessel formation, but perhaps also in the maintenance of the choriocapillaris. (Invest Ophthalmol Vis Sci. 2006;47:3135-3142) DOI:10.1167/iovs.051229

$\mathrm{T}$ he development of the eye, as other organs, depends on the concomitant formation of a complex vascular system to provide nutrients and oxygen. Thus, ocular development is associated with the formation of a vascular network that provides blood flow adapted to the physiologic needs of the mature retina. In most mammals, the adult retina is vascularized by two independent networks: the choroidal and the retinal vasculatures. During early development, the retina is oxygenated by the choroidal and hyaloid vessels. The vascularization of the retina itself occurs only during late gestation in humans and postnatally in rodents, with vessels restricted to

From the ${ }^{1}$ Schepens Eye Research Institute, the Departments of ${ }^{2}$ Ophthalmology and ${ }^{4}$ Pathology, and ${ }^{3}$ Department of Surgery, Children's Hospital, Harvard Medical School, Boston, Massachusetts.

Supported by National Eye Institute Grant EY015435 and National Cancer Institute Grant CA45548 (PAD).

Submitted for publication September 15, 2005; revised January 9 and February 28, 2006; accepted May 3, 2006.

Disclosure: M. Saint-Geniez, None; A.E. Maldonado, None; P.A. D'Amore, None

The publication costs of this article were defrayed in part by page charge payment. This article must therefore be marked "advertisement" in accordance with 18 U.S.C. $\$ 1734$ solely to indicate this fact.

Corresponding author: Patricia A. D'Amore, Schepens Eye Research Institute, 20 Staniford Street, Boston, MA 02114;

pdamore@vision.eri.harvard.edu. the inner retina. The outer retina remains completely avascular, which ensures visual clarity, and is nourished by the choroidal vasculature. The hyaloid vasculature meets the metabolic requirements of the developing lens but regresses during the final stage of ocular development. ${ }^{1}$

Formation of the choroidal vasculature is an early event that commences at the time of optic vesicle invagination when primary vessels originating from the perineural vascular plexus extend around the optic cup. This initial network expands into a complex vasculature with a layer of highly fenestrated capillaries, the choriocapillaris, underlying the outer retina. ${ }^{2}$ Although the molecular mechanisms governing the formation and survival of the choroid are unclear, the development of the choroidal vasculature appears to depend on the presence of differentiated RPE and its production of inductive signals.

Clinical observations and experimental models have shown that suppression of RPE differentiation inhibits choroidal development. $^{3-5}$ In addition, studies from animal models have demonstrated that destruction of the RPE leads to loss of fenestrations and atrophy of the underlying choroidal vessels, closely representing alterations observed in geographic atrophy. ${ }^{6,7}$ This crucial association between the RPE and choroidal vessels during development appears to persist in the adult. In age-related macular degeneration (ARMD), choriocapillaris atrophy is preceded by alterations in the RPE. 8,9

The paracrine interaction between the two tissues is also anatomically reflected by the polarization of both layers. The choriocapillaris is adjacent to the RPE layer and the choriocapillaris endothelial cells present fenestrations only on the side facing the RPE. Similarly, the RPE extends basal infoldings toward the choriocapillaris. Although numerous observations indicate that RPE has a trophic effect on the choroidal vasculature, the mechanisms mediating this relationship are not known. In vitro experiments have demonstrated that RPE cells produce several soluble growth factors, including basic fibroblast growth factor (bFGF), ${ }^{10}$ platelet-derived growth factor (PDGF)-B, ${ }^{11}$ and vascular endothelial growth factor (VEGF). ${ }^{12}$

VEGF is a good candidate for mediating RPE growth and survival effects on choroidal vessels. VEGF is an endothelial cell growth factor essential for normal vascular development, as mice with null or heterozygous deletions for VEGF are embryonic lethal. ${ }^{13,14}$ Alternative splicing of VEGF mRNA leads to three isoforms in mouse $(120,164 \text {, and } 188 \text { amino acids })^{15}$ and at least five different human isoforms, ${ }^{16}$ which differ in their ability to bind to heparan sulfate proteoglycans (HSPGs) and neuropilin. Thus, VEGF120 is freely diffusible, whereas VEGF188 remains bound to the cell surface and extracellular matrix (ECM). Finally, VEGF164 binds heparin with moderate affinity and therefore can be found both free and bound to the matrix.

Previous studies have indicated that VEGF is expressed in the correct temporal and spatial pattern to impact the choroid. In rodents and humans, VEGF is expressed by RPE during late choroidal development, whereas the VEGF receptors VEGFR1 and VEGFR2 are expressed by periocular mesenchymal cells adjacent to the RPE. ${ }^{4,17,18}$ In a coculture experiment, RPEsecreted VEGF was shown to stimulate tube formation by the underlying choroidal endothelial cells. ${ }^{19}$ Although VEGF ex- 
Table 1. Oligonucleotide Primer Sequences and Lengths of the Specific Amplification Products

\begin{tabular}{|c|c|c|c|}
\hline Gene & Forward Primer $\left(5^{\prime}-3^{\prime}\right)$ & Reverse Primer $\left(5^{\prime}-3^{\prime}\right)$ & $\begin{array}{l}\text { Product } \\
\text { Size } \\
\text { (bp) }\end{array}$ \\
\hline VEGFR1 & gagagcatctataaggcagcggatt & cacgtttacaatgagagtggcagtg & 456 \\
\hline VEGFR2 & tacacaattcagagcgatgtgtggt & ctggttcctccaatgggatatcttc & 499 \\
\hline Neuropilin-1 & tcaggaccatacaggagatgg & tgacatcccattgtgccaac & 619 \\
\hline Neuropilin-2 & agactaccaccccatatcccatgg & ctgccetggtcctcacggatg & 421 \\
\hline GAPDH & gtggcaaagtggagatggttgcc & gatgatgacccgtttggctcc & 291 \\
\hline
\end{tabular}

pression by RPE has been found to decrease with age, it persists in adult rats. ${ }^{17}$ Taken together, these observations suggest a role for VEGF in the development and maintenance of the choroidal vasculature. Consistent with this concept, RPE cells in vitro have been shown to secrete VEGF basally (toward the choriocapillaris) and VEGF receptors are expressed on the choroidal endothelium facing the RPE layer in human. ${ }^{20}$

Although much is known about the formation of the retinal vasculature, little is known about the mechanism of the development and maintenance of the choroidal vessels. The present study was designed to address the hypothesis that RPE-secreted VEGF is involved in the formation and maturation of the choroidal vessels and in the survival of the adult choriocapillaris. Such information is essential, not only to understand how the RPE may support normal choroidal function, but also to provide insight into the contribution of RPE-derived VEGF in ocular diseases, such as neovascular ARMD and geographic atrophy.

\section{Methods}

\begin{abstract}
Animals
Adult (6 - 8 weeks old) C57Bl/6 and C57Bl/6-C2J (albino C57Bl/6) mice (Jackson Laboratory, Bar Harbor, ME) and timed-pregnant Swiss-Webster and $\mathrm{C} 57 \mathrm{Bl} / 6$ VEGF-LacZ ${ }^{21}$ mice were used in the study. All animal experiments were conducted according to the ARVO Statement for the Use of Animals in Ophthalmic and Vision Research under protocols approved by the Schepens Eye Research Institute Animal Care and Use Committee (ACUC).
\end{abstract}

\section{Histochemistry and Immunohistochemistry}

$\boldsymbol{\beta}$-Galactosidase Histochemistry. VEGF expression was visualized in whole embryos (E10.5 and E13.5) and in cryosections of whole adult eyes from VEGF-LacZ mice. Embryos were fixed overnight at $4{ }^{\circ} \mathrm{C}$ in $4 \%$ paraformaldehyde in PBS. For cryosections, embryos were embedded in OCT compound (Sakura Finetechnical, Torrance, CA). Wholemounted embryos or cryosections were stained for LacZ using the in situ $\beta$-galactosidase staining kit according to the manufacturer's protocol (Stratagene, La Jolla, CA).

Immunohistochemistry. Air-dried cryosections (10 $\mu \mathrm{m})$ were pretreated with $0.3 \% \mathrm{H}_{2} \mathrm{O}_{2}$ in methanol for 30 minutes to block endogenous peroxidase activity. Primary antibodies used were rat anti-mouse CD31 (Pharmingen, San Diego, CA), rat anti-endomucin (a gift from Dietmar Vestweber, Max-Planck-Institute, Bad Nauheim, Germany), and rabbit anti-mouse VEGFR2 T1014 (a gift from Rolf Brekken, University of Texas Southwestern Medical Center, Dallas, TX). ${ }^{22,23}$ Antibodies were visualized with the avidin-biotin-peroxidase technique and 3-amino-9-ethylcarbazole (AEC) or 3,3'-diaminobenzidine (DAB) substrates (Vector ABC kit; Vector Laboratories, Burlingame, CA). In each experiment, a section was incubated with isotope matched IgG as a negative control. After mounting, the sections were visualized and photographed with a microscope (Axioskope Mot 2; Carl Zeiss Meditec, Inc., Dublin, CA).
For fluorescent immunodetection of $\beta$-galactosidase, cryosections were incubated with a polyclonal antibody against $\beta$-galactosidase (ICN Pharmaceuticals, Aurora, $\mathrm{OH}$ ) followed by a rhodamine-conjugated anti-rabbit antibody. Cell nuclei were identified by $4^{\prime}, 6^{\prime}$-diamino2-phenylindole (DAPI) labeling.

\section{RNA Analysis}

Standard RT-PCR Analysis. Tissues were dissected under RNase-free conditions from 8-week-old adult $\mathrm{C} 57 \mathrm{Bl} / 6$ mice. Total RNA was extracted (RNAqueousTM-4PCR kit; Ambion Inc., Austin, TX), according to the manufacturer's protocol. Residual DNA was removed by treatment with 1 unit DNase I (Ambion) at $37^{\circ} \mathrm{C}$ for 20 minutes. One microgram of RNA was reverse-transcribed in the presence of $500 \mathrm{ng}$ of oligo dT12-18 with reverse transcriptase (Superscript II; Invitrogen, Carlsbad, CA) in a $20-\mu \mathrm{L}$ reaction at $42^{\circ} \mathrm{C}$ for 50 minutes and digested with 2 units of RNase $\mathrm{H}$.

One microliter of cDNA was used as a template in a $25-\mu \mathrm{L}$ amplification mixture containing $200 \mathrm{mM}$ dNTPs, 1 U Taq DNA polymerase (Roche Diagnostics, Indianapolis, IN), and $0.2 \mu \mathrm{M}$ of the appropriate VEGF receptor primer pair (Table 1) and amplified for 35 cycles. Amplification products were separated by agarose gel electrophoresis, stained with ethidium bromide, and visualized by UV light.

Real-Time PCR Analysis. mRNA were purified as just described. One microgram of RNA was reverse transcribed as described, except $500 \mathrm{ng}$ of random hexamers was used. One twentieth of the total cDNA (50 ng of equivalent RNA) was used in each amplification reaction. VEGF isoforms were quantified (Prism 9700 Sequence Detection System; Applied Biosystems, Inc. [ABI], Foster City, CA) according to the manufacturer's instructions. Reactions were performed in $25 \mu \mathrm{L}$ with $0.3 \mu \mathrm{M}$ primers ${ }^{24}$ and master mix (SYBR Green Master mix; ABI). PCR cycles consisted of an initial denaturation step at $95^{\circ} \mathrm{C}$ for 10 minutes, followed by 40 cycles at $95^{\circ} \mathrm{C}$ for 15 seconds and at $60^{\circ} \mathrm{C}$ for 60 seconds. To confirm amplification specificity, PCR products from each primer pair were subjected to a melting curve analysis. Amplification of the $18 \mathrm{~S}$ RNA was performed on each sample (Eukaryotic $18 \mathrm{~S}$ rRNA Endogenous Control kit; ABI) as a control for sample loading and to allow normalization between samples. A standard curve was constructed for each PCR reaction and was derived from the serial dilution $\left(10^{-3}\right.$ to $10^{-9}$ ng DNA per reaction) of a plasmid coding for each isoform-VEGF188, VEGF164, and VEGF120 - and amplified using the SYBR Green system. The level of isoform expression in each sample was calculated relative to the standard curve. Each sample was run in triplicate, and each experiment included three nontemplate control wells. Results were expressed as the mean \pm standard deviation.

\section{Activation Status of VEGFR2}

Cell Lines and VEGF Treatment. Cultured porcine aortic endothelial cells overexpressing the human VEGFR2 (PAE-VEGFR2; a gift from Lena Claesson-Welsh; Ludwig Institute, Uppsala, Sweden) were maintained in DMEM supplemented with $10 \%$ fetal bovine serum and $2 \mathrm{mM}$ glutamine and antibiotics at $37^{\circ} \mathrm{C}$ and were grown in $5 \%$ $\mathrm{CO}_{2}$. To assay VEGFR2 phosphorylation, subconfluent cells were incubated overnight in serum-free medium and stimulated for 5 minutes with $50 \mathrm{ng} / \mathrm{mL}$ of recombinant VEGF165 (R\&D Systems, Minneapolis, $\mathrm{MN}$ ). Reactions were terminated by washing in cold PBS. 
FiguRE 1. Pattern of VEGF expression and localization of blood vessels during early eye development. Cryosections of E10.5 (A) and E13.5 (B, C, D) VEGF-LacZ/+ mouse were stained for $\beta$-galactosidase (blue) and $\mathrm{CD} 31$ (red). (A) At E10.5, VEGF was strongly expressed in the inner layer of the neural retinal and in the RPE cells. CD31-positive endothelial cells surround the optic cup. (B) At E13.5, VEGF expression in the retina was restricted to the RPE cells. High levels of VEGF expression was detected in the primary lens fibers and lens epithelium. (C) A higher power view of the RPE-choroid complex shows the RPE-specific expression of VEGF. The choriocapillaris and main choroidal vessels can be distinguished. (D) Serial section stained with nonimmune mouse IgG confirmed the specificity of CD31 antibody and the restriction of VEGF expression to the RPE layer. on, optic nerve; nr, neuronal retina; chd, choroid; cc, choriocapillaris; le, lens epithelium; plf, primary lens fiber; hv, hyaloid vessels; pm, pupillary membrane; tvl, tunica vasculosa lentis.
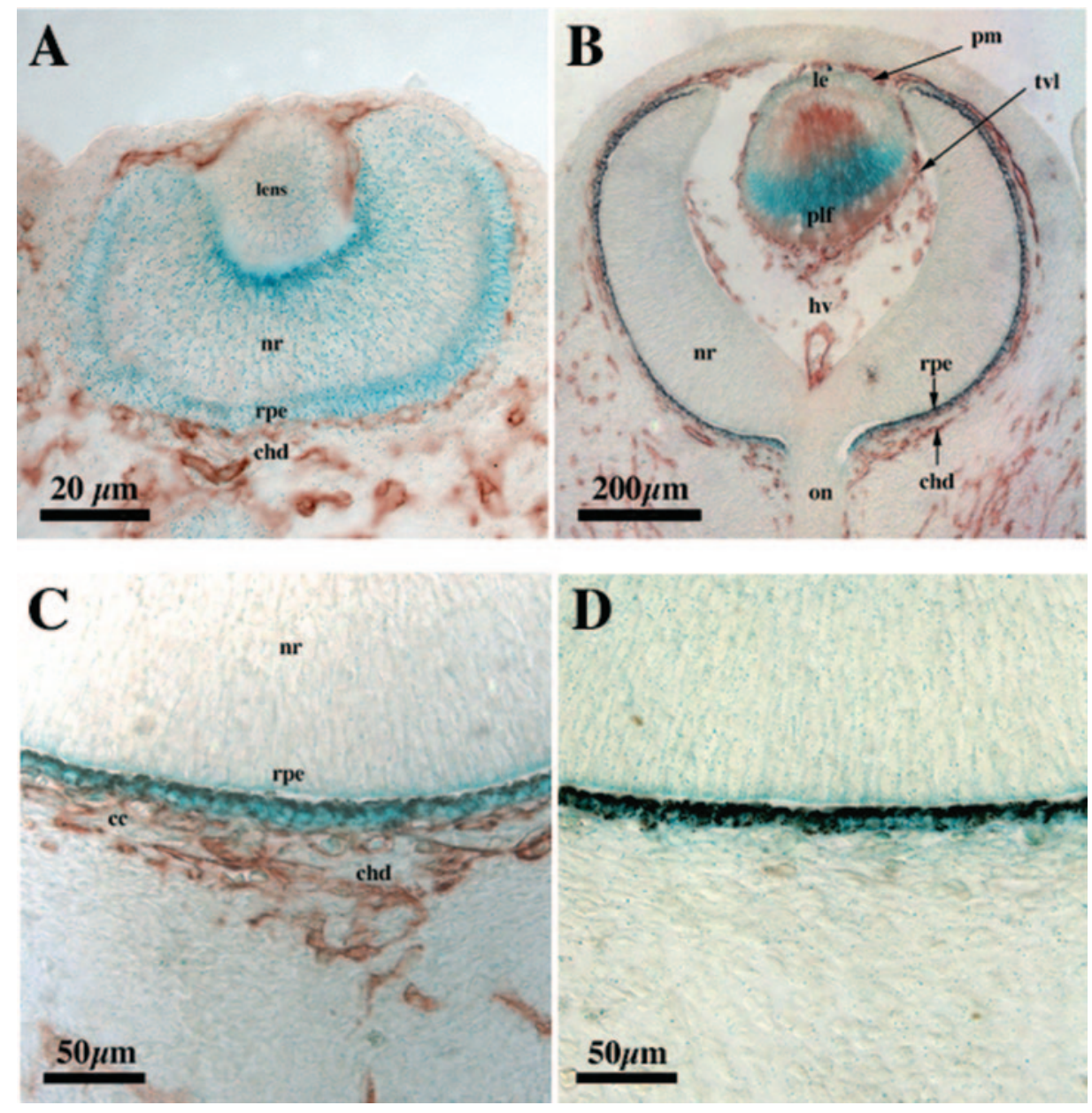

\section{D} $\checkmark$

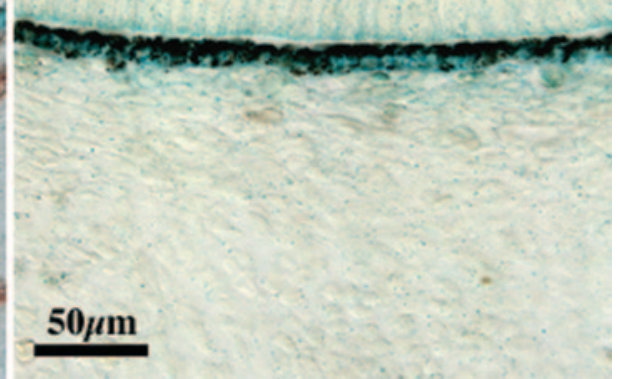

Immunoprecipitation and Western Blot Analysis. Tissues and cells were collected in lysis buffer (10 mM Tris-HCl [pH 7.4], $5 \mathrm{mM}$ EDTA, $50 \mathrm{mM} \mathrm{NaCl}, 1 \%$ Triton X-100, $50 \mathrm{mM} \mathrm{NaF}, 1 \mathrm{mM}$ phenylmethylsulfonyl fluoride (PMSF), $2 \mathrm{mM} \mathrm{Na}_{3} \mathrm{VO}_{4}$, and $20 \mathrm{mg} / \mathrm{mL}$ aprotinin). Proteins were quantified using a protein assay kit $\left(\mathrm{D}_{\mathrm{c}}\right.$; Bio-Rad, Hercules, CA). VEGFR2 was immunoprecipitated with a polyclonal rabbit anti-mouse VEGFR2 antibody (kind gift from Nader Rahimi, Boston University Medical Center) overnight at $4^{\circ} \mathrm{C}$. Immunoprecipitated complexes were analyzed by SDS-PAGE with a cocktail (1:1) of anti-phosphotyrosine antibodies: PY20 (Transduction Laboratories, Lexington, KY) and 4G10 (Upstate USA, Inc., Chicago, IL). The membranes were stripped by incubation for 30 minutes in $6.25 \mathrm{mM}$ Tris-HCl [pH 6.8], 2\% SDS, and $100 \mathrm{mM} \beta$-mercaptoethanol at $50^{\circ} \mathrm{C}$ and reprobed with a polyclonal VEGFR2 antibody (Santa Cruz Biotechnology, Santa Cruz, CA) to detect total VEGFR2.

\section{Results}

\section{Localization of VEGF and VEGFR2 during Early Eye Development}

To examine VEGF expression patterns in the developing eye, we used mice heterozygous for the LacZ reporter gene with a nuclear localization signal under the control of the VEGF promoter. $^{21}$ VEGF expression was determined by histochemical localization of $\beta$-galactosidase activity. RPE have been shown to express VEGF during development, but these studies were focused on at later stages of choroid development and used methods (in situ hybridization and immunohistochemistry) that do not allow clear determination of the cell types express- ing VEGF or its receptors. ${ }^{17,18}$ In contrast, we determined VEGF and VEGFR2 expression patterns at very early stages of choroidal development after invagination of the optic cup. At embryonic day (E)10.5, $\beta$-galactosidase activity, indicating VEGF expression, was detected in the primitive RPE cells. A second site of VEGF expression was localized to the inner nuclear layer (Fig. 1A). At this stage, $\beta$-galactosidase staining was weak and diffuse in the lens. Strong VEGF expression was detected in the posterior fibers of the lens at E12.5 (data not shown) and could be seen clearly at E13.5 (Fig. 1B). No VEGF was detected in the vitreous or associated with the hyaloid vessels (Fig. 1B). Higher power magnification of the back of the eye revealed strong VEGF expression restricted to the RPE (Figs. 1C, 1D). To visualize the developing vascular networks of the eye, we used the pan-endothelial cell (EC) marker CD31. At E10.5, CD31 immunolocalization revealed the presence of numerous ECs surrounding the posterior part of the retina (Fig. 1A). This primitive network differentiated rapidly into major vessels and smaller capillaries adjacent to the RPE (Fig. 1B, 1C).

To identify cells that would be targeted by VEGF, we determined VEGFR2 expression at the same developmental stages by using immunochemistry. At E10.5, VEGFR2 immunoreactivity was localized to the mesenchymal cells surrounding the optic cup. Comparison with CD31 staining (Fig. 1), shows that VEGFR2 is expressed by the ECs of the forming vascular beds, the choroid in the posterior part of the eye cup and the tunica vasculosa lentis (Fig. 2A). Although VEGFR2 has been thought to be specific for the hematopoietic lineage, VEGFR2 protein 

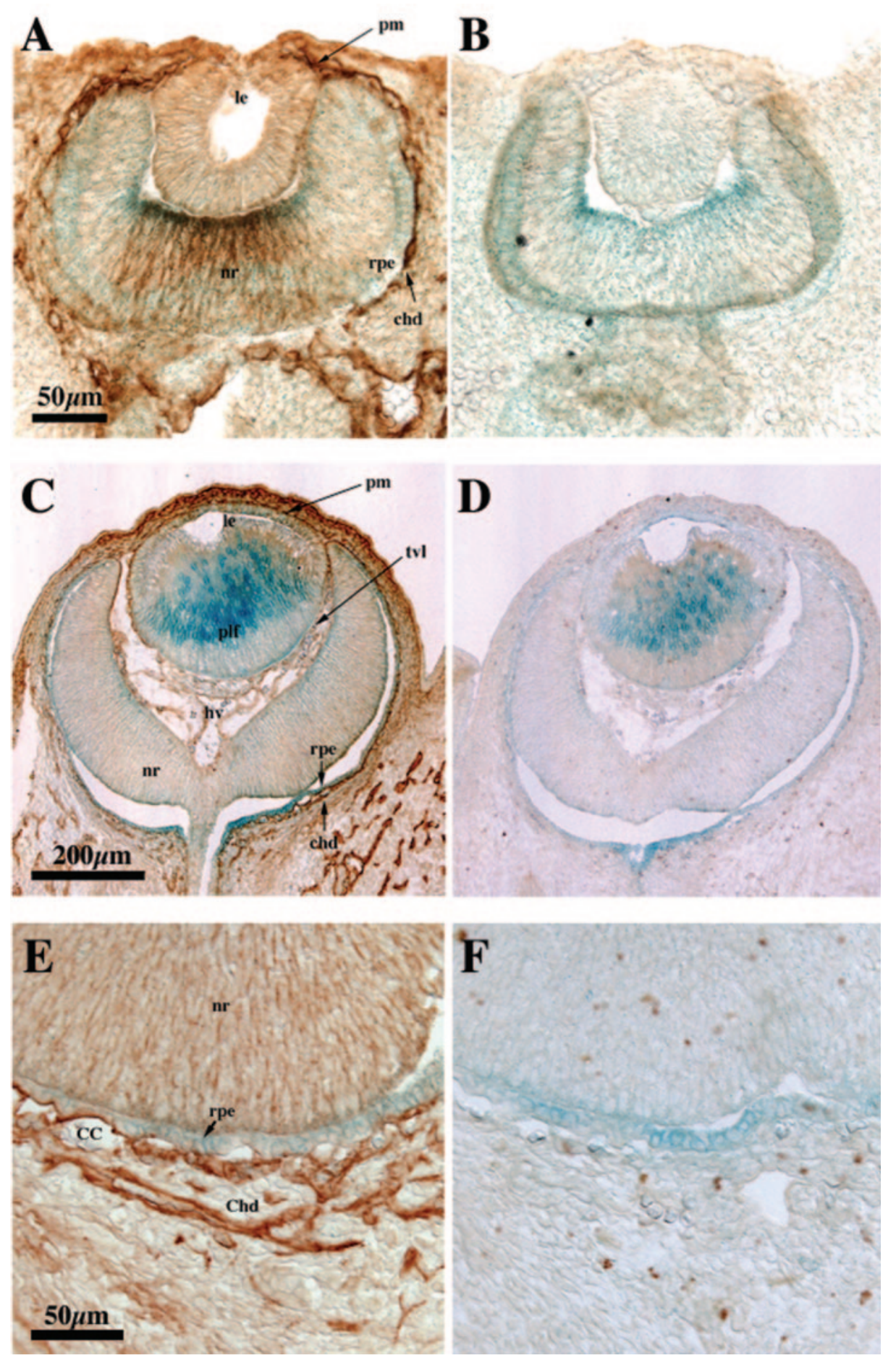

Figure 2. Expression of VEGFR2 during early eye development. Cryosections $(10 \mu \mathrm{m})$ of E10.5 (A, B) and E13.5 (B-F) VEGF-LacZ/+ mouse were stained for $\beta$-galactosidase (blue) and immunostained using a polyclonal antibody against VEGFR2 (A, C, E) or nonimmune rabbit IgG (B, D, F). (A) At E10.5, VEGFR2 expression was detected in the vascular network surrounding the optic cup, in the forming pupillary membrane and in the central part of the neural retina. (C) At E13.5, VEGFR2 labeling in the neuroblastic layers of the retina decreased, whereas it persisted in the forming choroidal vessels, hyaloid vessels, and tunica vasculosa lentis. (E) A higher magnification of the posterior part of the retina at E13.5 where forming choroidal vessels were highly positive for VEGFR2, with stronger staining in the ECs closely associated with the RPE laver. $(\mathbf{B}, \mathbf{D}, \mathbf{F})$ The use of rabbit IgG as control on serial sections confirms the specificity of the VEGFR2 antibody. on, optic nerve; nr, neuronal retina; chd, choroid; cc, choriocapillaris; le, lens epithelium; hv, hyaloid vessels; pm, pupillary membrane. was also detected in the central neural retina. Neural expression was weaker at E13.5 when VEGFR2 was restricted to the fetal vasculature and the forming choroid (Fig. 2B), particularly the developing choriocapillaris (Fig. 2C). No VEGFR2 was detected in the RPE layer at any developmental time point examined.

\section{RPE-Derived VEGF Expression in the Adult}

Assessment of VEGF expression in the adult RPE was complicated by the presence of pigment, which obscured visualization of $\beta$-galactosidase activity. Therefore, $\beta$-galactosidase was localized by fluorescence immunolabeling. Cryosections of adult mouse retinas stained for $\beta$-galactosidase revealed strong expression of VEGF in the RPE layer, whereas no expression was detected within the choroid or neural retina (Fig. 3A). We confirmed this result and provided conclusive demonstration of the lack of expression in the choroid by examining albino SW VEGF-LacZ mice (negative for the $r d$ mutation), which allowed us to detect VEGF expression by enzymatic $\beta$-galactosidase staining in the absence of interfering pigment. In adult albino mice, VEGF expression in the posterior part of the eye was clearly restricted to the RPE (Fig. 3B) and was entirely absent from the choroid, confirming our previous observations. 

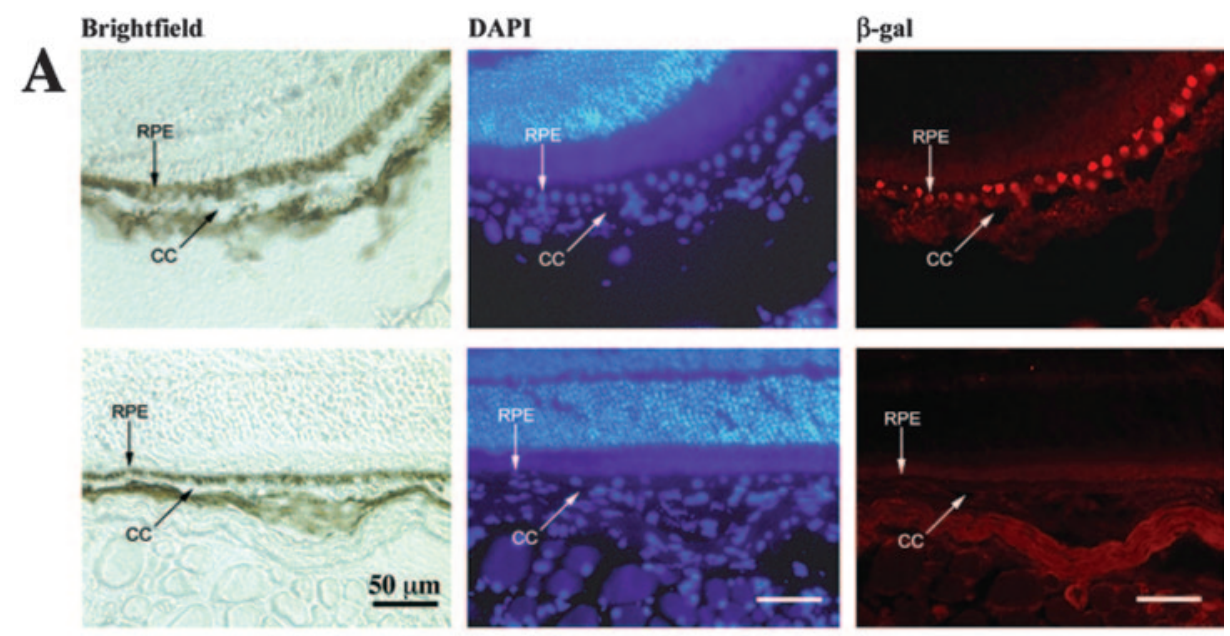

Figure 3. RPE-expression of VEGF in adult mouse. (A) Cryosections of adult $\mathrm{C} 57 \mathrm{Bl} / 6 \mathrm{~J}$ VEGF-LacZ mouse eyes immunostained for $\beta$-galactosidase $(t o p)$ revealed strong VEGF expression in the RPE layer and no expression within the choroid or the neural retina. Nuclei were identified by DAPI staining. Bottom: nonimmune rabbit IgG yielded no signal. (B) The previous result is confirmed in adult SW VEGF-LacZ mouse eye section stained for $\beta$-galactosidase activity. ONL, outer nuclear layer; OS, outer segment; Chd, choroid; CC, choriocapillaris.

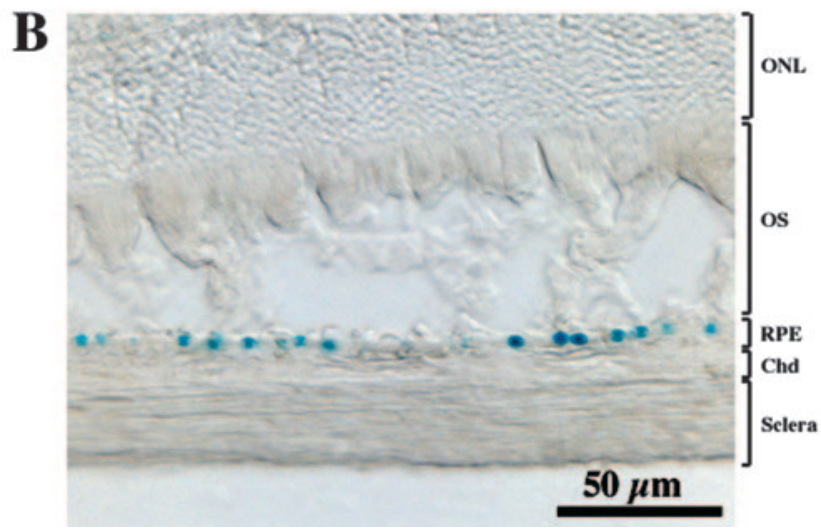

VEGF isoform expression in freshly dissected adult tissues was assessed by real-time PCR. To avoid artifacts caused by RPE isolation and because RPE is the only source of VEGF in the posterior part of the adult eye (Fig. 3), RNA was directly purified from the entire back layer of the mouse eye (choroid and RPE). We have shown that total VEGF expression and relative isoform distribution differ among tissues. ${ }^{25}$ Adult lung and retina displayed high levels of VEGF mRNA (Fig. 4A). Compared with the brain, RPE represented a site of strong VEGF expression. VEGF164 was the most abundant isoform detected in most of the organs examined, with the exception of the lung, which expressed predominantly ( 70\%) VEGF 188 (Fig. 4B). Of note, the RPE expressed only the diffusible isoforms, VEGF164 and VEGF120 (75\% and 24\%, respectively), whereas the cell-associated VEGF188 was almost undetectable (1\%).

\section{Expression of VEGF Receptors and VEGFR2 Activation Status in Adult Choroid}

To investigate further the relationship between RPE-secreted VEGF and the choroid, we examined the expression of VEGF receptors and coreceptors in the choroid by RT-PCR. VEGFR1, VEGFR2, and coreceptors, neuropilin-1 and -2, were expressed in the adult choroid-RPE complex (Fig. 5). Next, we examined the localization of VEGFR2 protein by immunohistochemistry. Consistent with RT-PCR, VEGFR2 was strongly expressed by EC of the choroidal vessels. Whereas no VEGFR2 was detected in developing RPE (Fig. 2), VEGFR2 expression was clearly seen in adult RPE, although the labeling in the RPE was lighter than in the choroid. More specifically, VEGFR2 seemed to be preferentially expressed in the choriocapillaris adjacent to the
RPE layer, with significantly less VEGFR2 staining in the major vessels of the choroid (Fig. 6C). To confirm that this difference in staining between capillaries and main vessels was specific for VEGFR2, we used two different endothelium-specific markers, endomucin and CD31. Endomucin is an endotheliumspecific sialomucin that has been shown to be expressed in all endothelia as early as E8.0. ${ }^{26,27}$ Staining of adult mouse choroid revealed that endomucin was highly expressed in all the choroidal ECs (Fig. 6A) whereas CD31 was detected in choriocapillaris and some large choroidal vessels (Fig. 6B). Comparison of VEGFR2 expression pattern to endomucin and CD31 staining clearly demonstrated that VEGFR2 was selectively expressed by the endothelium of choriocapillaris facing the RPE layer.

However, the presence of VEGF receptors does not prove that VEGF is actively signaling. To address this question, we investigated the activation status of VEGFR2 in the adult choroid. This was accomplished by immunoprecipitation of adult murine and adult bovine choroid lysates with anti-VEGFR2 antisera followed by Western blot analysis with antisera against phosphotyrosine. Results revealed that VEGFR2 is activated in adult mouse and bovine choroid (Fig. 7). VEGFR2 activation in the adult choroid may be unexpected, since the choroidal vasculature is mature and proliferation has ceased.

\section{Discussion}

To assess the potential role of VEGF in the paracrine interaction between the RPE and the choroid, we have examined the expression patterns of VEGF and VEGFR2 in early eye development and in the adult. Growth factors involved in the de- 
A

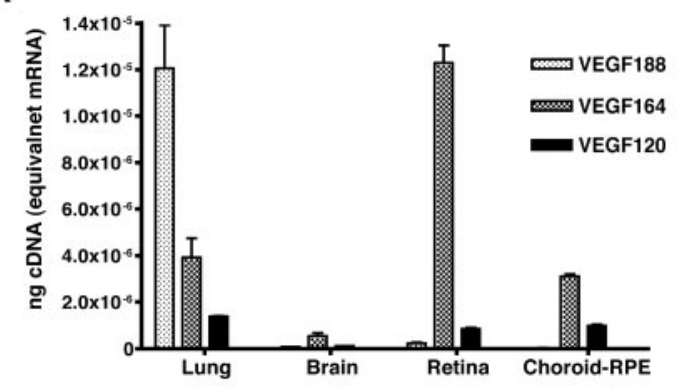

B

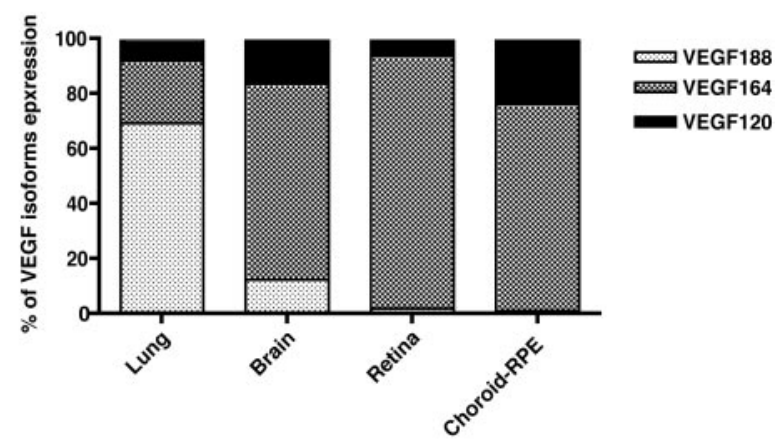

FIGURE 4. Quantification of VEGF isoform expression in adult tissues. (A) Levels of VEGF isoform mRNA were measured by real-time PCR in lung, brain, retina, and choroid-RPE complex. The amount of cDNA was quantified with a standard curve corrected for the $18 \mathrm{~S}$ content of the samples. VEGF isoforms were found to be differentially expressed. The lung and the retina showed the highest overall VEGF mRNA expression, with lung expressing primarily VEGF188 and retina VEGF164. In the choroid-RPE complex, only the isoforms 120 and 164 were expressed. (B) Percentile representation of the isoforms expression with total VEGF set to $100 \%$. Choroid-RPE is the site of highest expression of VEGF120 and lowest expression of VEGF188.

velopment of the choroid have been largely unstudied. Although numerous studies have shown that RPE in culture synthesize and secrete $\mathrm{VEGF}^{12,20}$ there are limited reports of VEGF expression by adult RPE in the absence of disease. Cells in culture often synthesize growth factors that they either do not produce in vivo or make only during development or wound healing. Thus, the ability of RPE to make VEGF in culture does not provide reliable insight into VEGF expression by RPE in adults. Using VEGF-LacZ mice we have clearly demonstrated that RPE begin to express VEGF at the earliest stages of eye formation, just after invagination of the optic cup. At this time, VEGFR2 is expressed by mesenchymal cells, presumably endothelial cell progenitors, surrounding the optic cup. CD31 staining revealed that the initial maturation of the choroid, the organization into choriocapillaris closely associated with the RPE and larger choroidal vessels, occurs at the beginning of formation of the eye vasculature at E13.5. VEGF involvement in this process is strongly suggested by the pattern of its expression and that of its main receptor, VEGFR2. Although one of VEGF's major functions is to increase endothelial cells permeability, in part by the induction of fenestrations, ${ }^{28,29}$ its role in the acquisition of the fenestrated phenotype remains to be investigated.

RPE-secreted VEGF may also influence neural development. VEGFR2 has been shown to be expressed by neural progenitors of the developing retina (this article and Ref. 30). It is noteworthy that at E10.5 VEGF expression can also be detected in the inner part of the forming retina. In addition, VEGF has recently been revealed as a neuroprotective and neurogenesis factor. ${ }^{31}$ Therefore, neural retina and RPE-derived VEGF could also be involved in the proliferation and/or differentiation of neuronal cells of the retina. The critical role of RPEsecreted VEGF in the formation of choroidal vessels has been recently investigated by using cre-lox strategy to inactivate specifically VEGF expression in the RPE cells. As expected, the early inactivation of VEGF expression led to the almost complete absence of choroidal vessels, microphthalmia, and loss of vision. ${ }^{32}$ Although this model confirms that RPE-secreted VEGF is necessary for choroid formation, the early inactivation of the VEGF gene (E11.5) at the time of endothelial cell recruitment and proliferation does not address the function of VEGF at later stages of choroidal vessel development or in the adult.

We have shown that RPE expression of VEGF does not cease with the maturation of the choroidal vasculature, but continues in the adult in the absence of angiogenesis or disease. Expression of VEGF persists in most adult organs where VEGF is frequently produced by epithelial cells adjacent to a microvascular bed where the endothelium expresses VEGF receptors. Examples of this coordinate expression pattern include the lung, where VEGF is produced by pulmonary epithelium just next to the alveolar endothelium; the kidney, where VEGF is expressed by podocytes near the glomerular capillaries $^{33}$; and the eye, where pericytes and astrocytes adjacent to retinal vessels express VEGF. ${ }^{34}$ However, the level of VEGF expression and distribution of isoforms differs significantly among organs (this article and Ref. 35). Our results indicate that RPE express the diffusible isoforms, VEGF164 and VEGF120, but not VEGF188, the cell-associated form. This finding is compatible with a function of VEGF as a survival factor for the choroid where it would need to diffuse through Bruch's membrane to access the choriocapillaris. In contrast,
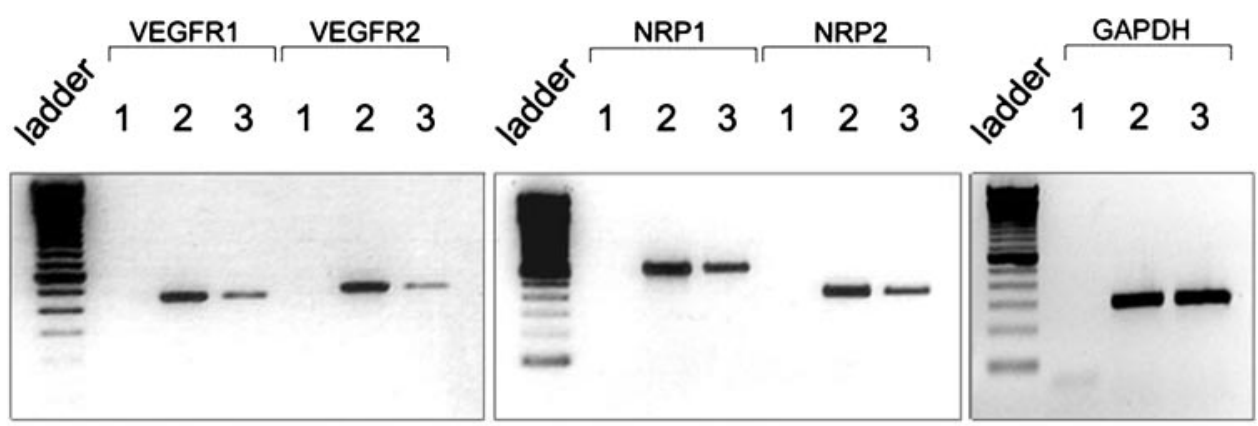

\section{1: PCR control \\ 2: lung \\ 3: choroid+RPE}

FIGURE 5. Expression of VEGF receptors and coreceptors in the choroid-RPE complex. RT-PCR revealed the expression of VEGFR1, VEGFR2, NRP1, and NRP2 in choroid-RPE tissue from 8-week-old mice. cDNA from lung was included as a positive control. PCR reactions without cDNA are presented as negative control. 
FIGURE 6. Localization of endomucin, CD31, and VEGFR2 in adult choroid. (A) Endomucin was strongly expressed on all endothelium of the choriocapillaris and choroid. (B) CD31 expression was limited to the choriocapillaris and some main vessels of the choroid. (C) By comparison to endomucin and CD31 staining, VEGFR2 expression was at particularly high levels in the choriocapillaris whereas no major vessels of the choroid were stained. (D) Negative control. Chd, choroid; CC, choriocapillaris. Scale bar, $20 \mu \mathrm{m}$.
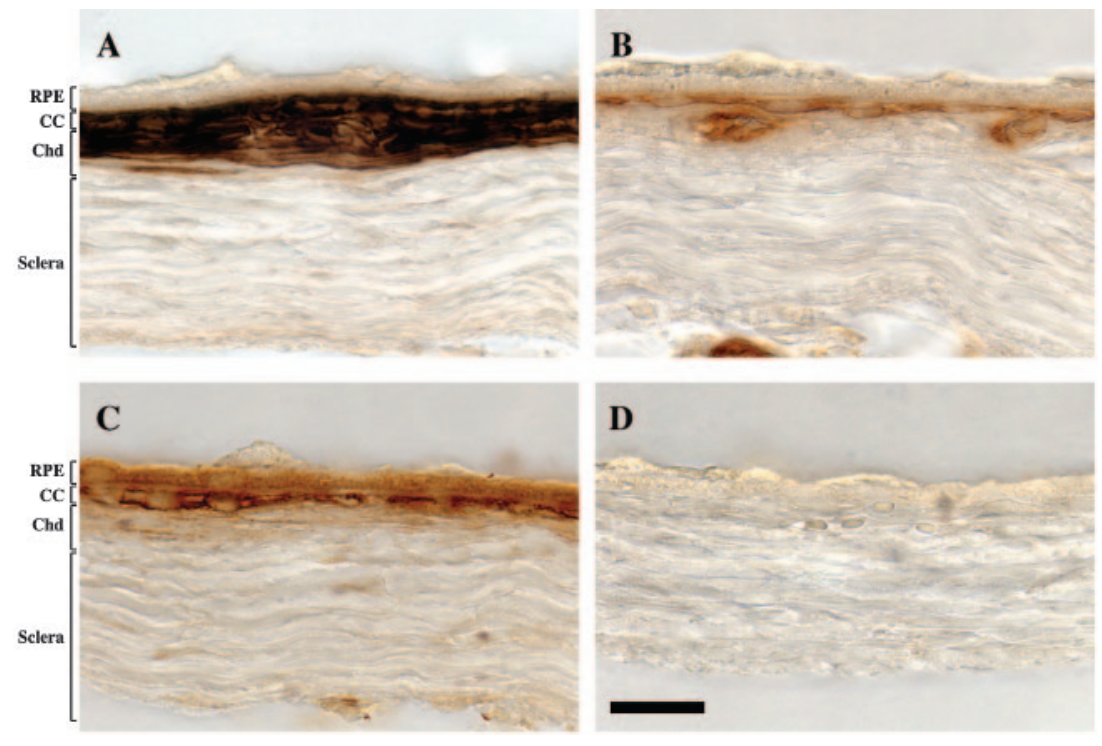

VEGF188 is the predominant isoform produced in the lung, where epithelium-secreting VEGF is in very close contact with the alveolar microvessels. 35

One surprising finding is that VEGFR2 was expressed in adult RPE, whereas no expression was detected in embryonic RPE. To our knowledge, no VEGFR2 staining in adult retina has been reported but such analyses have been limited by the quality of the antibodies used and the presence of the pigment. VEGFR2 mRNA has been reported in adult human RPE in vivo. ${ }^{36}$ Our findings that adult RPE express both VEGF and VEGFR2 suggest that there may be an autocrine loop for VEGF in vivo, an observation that had been shown for cultured RPE. $^{37}$

In support of the fact that RPE-secreted VEGF diffuses through Bruch's membrane and reaches the underlying choriocapillaris, in our study VEGFR2 was activated in adult choroid. In the adult, where this signaling does not lead to vessel growth, we hypothesize that it instead reflects the survival role that VEGF plays in the adult vasculature. A survival role for VEGF in quiescent endothelium has been suggested in studies in which anti-VEGFR2 molecules were used. Administration of the VEGFR2-selective tyrosine kinase inhibitor SU5416 to adult

\section{PAE-VEGFR2 Choroids}

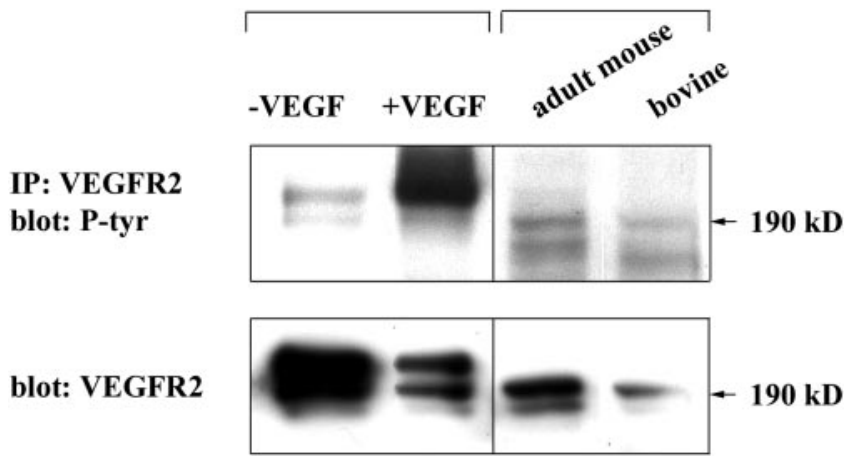

FIGURE 7. VEGFR2 activation status in the choroid. VEGFR2 lysates of adult murine and adult bovine choroid lysates were immunoprecipitated with anti-VEGFR2 antisera and then Western blotted with antisera against phosphotyrosine. PAE-VEGFR2 cells, untreated or treated with VEGF, were used as the positive control. Results revealed that VEGFR2 is constitutively phosphorylated in both adult mouse and bovine choroids. rats led to alveolar cell apoptosis as a consequence of capillary endothelial cells death in the lung. ${ }^{38}$ Significant capillary loss was also observed in the trachea after systemic treatment of adult mice with VEGF-Trap. ${ }^{39}$

The dependence of the choroidal vasculature on the RPE is reflected by the disease and abnormalities observed when the RPE layer is not able to differentiate properly ${ }^{4,40}$ or is damaged. $^{6,7}$ For example, the advanced form of geographic atrophy or dry age-related macular degeneration is characterized by a localized degeneration of the RPE, resulting in the atrophy of the underlying choriocapillaris. Loss of VEGF expression by the abnormal RPE layer could explain the endothelial apoptosis observed. Taken together, our data provide strong support for our hypothesis that RPE-secreted VEGF constitutively signals choroidal endothelial cells in vivo. We speculate that this paracrine communication mediates the maintenance of a normally functioning, fenestrated, quiescent choriocapillaris in the adult.

\section{Acknowledgments}

The authors thank Kameran Lashkari for technical advice, Andras Nagy for the VEGF-LacZ mice, and Diane Darland for helpful discussions and encouragement.

\section{References}

1. Barishak YR. Embryology of the eye and its adnexae. Dev Ophthalmol. 1992;24:1-142.

2. Saint-Geniez M, D'Amore PA. Development and pathology of the hyaloid, choroidal and retinal vasculature. Int J Dev Biol. 2004;48: 1045-1058.

3. Torczynski E. Choroid and suprachoroid. In: Jakobiec FA, ed. Ocular Anatomy, Embryology and Teratology. Philadelphia: Harper \& Row; 1982:553-585.

4. Zhao S, Overbeek PA. Regulation of choroid development by the retinal pigment epithelium. Mol Vis. 2001;7:277-282.

5. Rousseau B, Dubayle D, Sennlaub F, et al. Neural and angiogenic defects in eyes of transgenic mice expressing a dominant-negative FGF receptor in the pigmented cells. Exp Eye Res. 2000;71:395404 .

6. Korte GE, Reppucci V, Henkind P. RPE destruction causes choriocapillary atrophy. Invest Ophthalmol Vis Sci. 1984;25:1135-1145.

7. Del Priore LV, Hornbeck R, Kaplan HJ, et al. Debridement of the pig retinal pigment epithelium in vivo. Arch Opbthalmol. 1995; 113:939-944. 
8. Sarks SH. Ageing and degeneration in the macular region: a clinicopathological study. Br J Ophthalmol. 1976;60:324-341.

9. Young RW. Pathophysiology of age-related macular degeneration. Surv Ophthalmol. 1987;31:291-306.

10. Bost LM, Aotaki-Keen AE, Hjelmeland LM. Coexpression of FGF-5 and bFGF by the retinal pigment epithelium in vitro. Exp Eye Res. 1992;55:727-734.

11. Handa JT, Reiser KM, Matsunaga H, Hjelmeland LM. The advanced glycation endproduct pentosidine induces the expression of PDGF-B in human retinal pigment epithelial cells. Exp Eye Res. 1998;66:411- 419 .

12. Adamis AP, Shima DT, Yeo KT, et al. Synthesis and secretion of vascular permeability factor/vascular endothelial growth factor by human retinal pigment epithelial cells. Biochem Biophys Res Commun. 1993;193:631-638.

13. Carmeliet P, Ferriera V, Breier G, et al. Abnormal blood vessel development and lethality in embryos lacking a single VEGF allele. Nature. 1996;380:435-439.

14. Ferrara N, Carver-Moore K, Chen H, et al. Heterozygous embryonic lethality induced by targeted inactivation of the VEGF gene. $\mathrm{Na}$ ture. 1996;380:439-442.

15. Shima DT, Kuroki M, Deutsch U, et al. The mouse gene for vascular endothelial growth factor: genomic structure, definition of the transcriptional unit, and characterization of transcriptional and post-transcriptional regulatory sequences. J Biol Chem. 1996;271: 3877-3883.

16. Houck KA, Ferrara N, Winer $\mathrm{J}$, et al. The vascular endothelial growth factor family: identification of a fourth molecular species and characterization of alternative splicing of RNA. Mol Endocrinol. 1991;5:1806-1814.

17. Yi X, Mai LC, Uyama M, Yew DT. Time-course expression of vascular endothelial growth factor as related to the development of the retinochoroidal vasculature in rats. Exp Brain Res. 1998; 118:155-160.

18. Gogat K, Le Gat L, Van Den Berghe L, et al. VEGF and KDR gene expression during human embryonic and fetal eye development. Invest Ophthalmol Vis Sci. 2004;45:7-14.

19. Sakamoto T, Sakamoto H, Murphy TL, et al. Vessel formation by choroidal endothelial cells in vitro is modulated by retinal pigment epithelial cells. Arch Ophthalmol. 1995;113:512-520.

20. Blaauwgeers HG, Holtkamp GM, Rutten $H$, et al. Polarized vascular endothelial growth factor secretion by human retinal pigment epithelium and localization of vascular endothelial growth factor receptors on the inner choriocapillaris: evidence for a trophic paracrine relation. Am J Pathol. 1999;155:421-428.

21. Miquerol L, Gertsenstein M, Harpal K, Rossant J, Nagy A. Multiple developmental roles of VEGF suggested by a LacZ-tagged allele. Dev Biol. 1999;212:307-322.

22. Feng D, Nagy JA, Brekken RA, et al. Ultrastructural localization of the vascular permeability factor/vascular endothelial growth factor (VPF/VEGF) receptor-2 (FLK-1, KDR) in normal mouse kidney and in the hyperpermeable vessels induced by VPF/VEGF-expressing tumors and adenoviral vectors. J Histochem Cytochem. 2000;48: 545-556.

23. Brekken RA, Overholser JP, Stastny VA, et al. Selective inhibition of vascular endothelial growth factor (VEGF) receptor 2 (KDR/Flk-1) activity by a monoclonal anti-VEGF antibody blocks tumor growth in mice. Cancer Res. 2000;60:5117-5124.
24. Zhang L, Conejo-Garcia JR, Yang N, et al. Different effects of glucose starvation on expression and stability of VEGF mRNA isoforms in murine ovarian cancer cells. Biochem Biophys Res Commun. 2002;292:860-868.

25. Ng YS, D'Amore PA. Therapeutic angiogenesis for cardiovascular disease. Curr Control Trials Cardiovasc Med. 2001;2:278-285.

26. Brachtendorf G, Kuhn A, Samulowitz U, et al. Early expression of endomucin on endothelium of the mouse embryo and on putative hematopoietic clusters in the dorsal aorta. Dev Dyn. 2001;222: $410-419$.

27. Morgan SM, Samulowitz U, Darley L, Simmons DL, Vestweber D. Biochemical characterization and molecular cloning of a novel endothelial-specific sialomucin. Blood. 1999;93:165-175.

28. Roberts WG, Palade GE. Increased microvascular permeability and endothelial fenestration induced by vascular endothelial growth factor. J Cell Sci. 1995;108:2369-2379.

29. Yokomori H, Oda M, Yoshimura K, et al. Vascular endothelial growth factor increases fenestral permeability in hepatic sinusoidal endothelial cells. Liver Int. 2003;23:467-475.

30. Yang K, Cepko CL. Flk-1, a receptor for vascular endothelial growth factor (VEGF), is expressed by retinal progenitor cells. J Neurosci. 1996;16:6089-6099.

31. Yasuhara T, Shingo T, Kobayashi K, et al. Neuroprotective effects of vascular endothelial growth factor (VEGF) upon dopaminergic neurons in a rat model of Parkinson's disease. Eur J Neurosci. 2004; 19:1494-1504.

32. Marneros AG, Fan J, Yokoyama Y, et al. Vascular endothelial growth factor expression in the retinal pigment epithelium is essential for choriocapillaris development and visual function. Am J Pathol. 2005;167:1451-1459.

33. Kitamoto Y, Tokunaga H, Tomita K. Vascular endothelial growth factor is an essential molecule for mouse kidney development: glomerulogenesis and nephrogenesis. J Clin Invest. 1997;99:23512357.

34. Darland DC, Massingham LJ, Smith SR, et al. Pericyte production of cell-associated VEGF is differentiation-dependent and is associated with endothelial survival. Dev Biol. 2003;264:275-288.

35. Ng YS, Rohan R, Sunday ME, Demello DE, D'Amore PA. Differential expression of VEGF isoforms in mouse during development and in the adult. Dev Dyn. 2001;220:112-121.

36. Chen YS, Hackett SF, Schoenfeld CL, et al. Localisation of vascular endothelial growth factor and its receptors to cells of vascular and avascular epiretinal membranes. Br J Ophthalmol. 1997;81:919926.

37. Guerrin M, Moukadiri H, Chollet P, et al. Vasculotropin/vascular endothelial growth factor is an autocrine growth factor for human retinal pigment epithelial cells cultured in vitro. J Cell Physiol. 1995;64:385-394.

38. Kasahara Y, Tuder RM, Taraseviciene-Stewart L, et al. Inhibition of VEGF receptors causes lung cell apoptosis and emphysema. J Clin Invest. 2000;106:1311-1319.

39. Baffert F, Thurston G, Rochon-Duck M, et al. Age-related changes in vascular endothelial growth factor dependency and angiopoietin-1-induced plasticity of adult blood vessels. Circ Res. 2004;94: 984-992.

40. Rousseau B, Larrieu-Lahargue F, Bikfalvi A, et al. Involvement of fibroblast growth factors in choroidal angiogenesis and retinal vascularization. Exp Eye Res. 2003;77:147-156. 